\title{
Ultra performance liquid chromatography-tandem mass spectrometry for the determination of epirubicin in human plasma
}

\author{
Ruiping Li ${ }^{\mathrm{a}, \mathrm{b}}$, Lili Dong ${ }^{\mathrm{a}, \mathrm{b}}$, Junxiong Huang ${ }^{\mathrm{a}, \mathrm{b}, *}$ \\ a State Key Laboratory of Environmental Chemistry and Ecotoxicology, Research Center for Eco-Environmental Sciences, \\ Chinese Academy of Sciences, P.O. Box 2871, Beijing 100085, China \\ ${ }^{\mathrm{b}}$ Graduate School of the Chinese Academy of Sciences (GSCAS), Beijing 100049, China
}

Received 23 March 2005; received in revised form 25 April 2005; accepted 26 April 2005

Available online 22 June 2005

\begin{abstract}
A novel method has been developed for the determination of epirubicin in human plasma by ultra performance liquid chromatography combined with tandem mass spectrometry (UPLC-MS/MS). Epirubicin and internal standard epidaunorubicin were achieved from plasma via solid-phase extraction (SPE) using Oasis HLB cartridge. The analysis was performed on an AcQuity UPLC ${ }^{\mathrm{TM}} \mathrm{BEH} \mathrm{C}_{18}$ column $(1.7 \mu \mathrm{m}$, $50 \mathrm{~mm} \times 1 \mathrm{~mm}$ i.d.) utilizing a gradient elution profile and a mobile phase consisting of $0.1 \%$ formic acid in water and acetonitrile. The analytes were detected using an electrospray ionization tandem mass spectrometry in positive ion mode with multiple reaction monitoring (MRM). This method combines both advantages of UPLC and MS/MS, producing superior reliability, sensitivity and accuracy to previously published methods. The calibration curve was linear $\left(r^{2}=0.998\right)$ over the concentration range of $0.50-100.0 \mathrm{ng} / \mathrm{ml}$. The limits of detection (LOD) and quantification (LOQ) for epirubicin were 0.10 and $0.50 \mathrm{ng} / \mathrm{ml}$ using $0.2 \mathrm{ml}$ plasma sample, respectively. Recoveries of greater than $89 \%$ with intra- and inter-day precision (R.S.D.) less than $12 \%$ were obtained at concentrations above the LOQ. The present method has been successfully applied to analyze human plasma samples taken from patients administered epirubicin intravenously. Also, the principal metabolite epirubicinol was detected in all the patient plasma samples under investigation. The proposed method is very rapid, reliable and sensitive, and can be applicable to therapeutical drug monitoring and pharmacokinetic studies of epirubicin.
\end{abstract}

(C) 2005 Elsevier B.V. All rights reserved.

Keywords: Epirubicin; Biosample analysis; Human plasma; Method validation; UPLC-MS/MS

\section{Introduction}

Anthracyclines, an important group of antibiotics, have been used in cancer chemotherapy for more than 30 years, and considered to be among the most useful antineoplastics. Toxic effects of the anthracyclines include myelosuppression, stomatitis, and nausea, etc.; but the most severe toxic effect is a cumulative dose-related cardiotoxicity [1]. Many efforts are now ongoing to attenuate the toxic effects by using combination chemotherapy regimen or varying schedule of administration [2,3]. These studies have increased the demand for fast and robust analytical method for moni-

\footnotetext{
* Corresponding author. Tel.: +86106294 3703; fax: +861062849337. E-mail address: junxionghuang@yahoo.com (J. Huang).
}

toring the actual concentration of the drugs in biological samples.

Among many hundred anthracyclines discovered and developed, epirubicin (EPI) is one of the most widely used in clinical practice due to its good therapeutic efficacy and low cardiotoxicity [1]. One important route of metabolic transformation of EPI in animals and man is the reduction of the side chain carbonyl group to the secondary alcohol epirubicinol (EPL). Up to now, several analytical methods for the determination of EPI and its metabolites have been developed, including capillary electrophoresis (CE) with laser-induced fluorescence detection [4] and high performance liquid chromatography (HPLC) with electrochemical [5], fluorometric [6-8], or mass spectrometric (MS) detections [9]. Methods using non-mass spectrometric detection may not have the 
desired selectivity and sensitivity [1,10,11]. Method based on a combination of liquid chromatography with mass spectrometry (LC-MS) could be a more reliable approach for the determination of drug at the lower concentration levels (ng/ml) but showed long run time [9]. The introduction of tandem mass spectrometric detection (MS/MS) has led to a significant improvement in detectability and selectivity by employing multiple reaction monitoring (MRM) data acquisition. However, there are no methods available for determination of EPI in plasma by LC-MS/MS.

The aim of this study was to develop a more rapid, sensitive and specific technique for the determination of EPI in human plasma by ultra performance liquid chromatography-tandem mass spectrometry (UPLC-MS/MS). Solid-phase extraction (SPE) using Oasis HLB cartridge was applied to extract the analytes in human plasma prior to UPLC-MS/MS analysis. UPLC employs $1.7 \mu \mathrm{m}$ particles, resulting in a very flat Van Deemter plot and a linear velocity faster than usual one with $5 \mu \mathrm{m}$ packings; consequently, improves resolution, speed and sensitivity for many HPLC methods. UPLC-MS/MS takes both advantages of the chromatographic benefits afforded by sub- $2 \mu \mathrm{m}$ particles and the high degree of accuracy, selectivity and specificity of the MS/MS detection. The established method has achieved both the desired sensitivity $(0.50 \mathrm{ng} / \mathrm{ml}$ using $0.2 \mathrm{ml}$ human plasma) and a fast run time ( $<4 \mathrm{~min}$ ), and has been validated in patients treated with EPI for lymphoma.

\section{Experimental}

\subsection{Materials and reagents}

Epirubicin (EPI) and epidaunorubicin (EPR), an internal standard (IS) for EPI as pure standards were kindly supplied by Hisun Pharmaceutical Inc. (Zhejiang, China) and stored at $+4{ }^{\circ} \mathrm{C}$ in the dark (Fig. 1). Solvents used for UPLC-MS/MS measurements were of HPLC grade, and all other reagents were of analytical grade. Deionized water was obtained from a Milli-Q system, Millipore (Brussels, Belgium). Oasis HLB $1 \mathrm{cc} / 30 \mathrm{mg}$ extraction cartridges (Part No. WAT094225) were provided by Waters (Milford, MA, USA). Control blank human plasma was supplied by healthy donors.

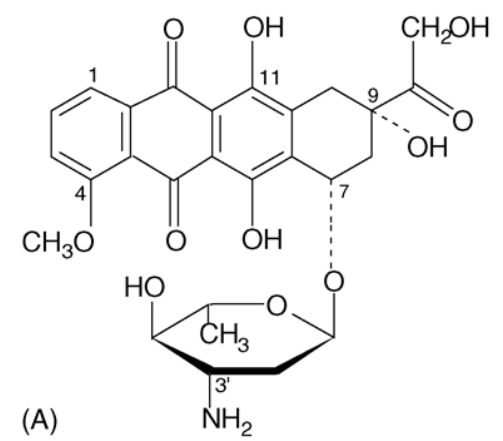

\subsection{Apparatus and operating conditions}

The UPLC-MS/MS system consisted of an AcQuity ${ }^{\mathrm{TM}}$ ultra performance liquid chromatograph and a Quattro Premier Micromass ${ }^{\circledR}$ mass spectrometer (Waters/Micromass, Milford, MA). An AcQuity UPLC ${ }^{\mathrm{TM}}$ BEH $\mathrm{C}_{18}$ column $(1.7 \mu \mathrm{m}, 50 \mathrm{~mm} \times 1 \mathrm{~mm}$ i.d.) also from Waters was used for the analysis. The column was maintained at $30^{\circ} \mathrm{C}$. The standards and sample extracts were chromatographed by UPLC with a gradient mobile phase consisting of $0.1 \%$ formic acid in water as solvent A and acetonitrile (ACN) as solvent B. The gradient condition of mobile phase is: $0 \mathrm{~min} 85 \% \mathrm{~A}$, $1.00 \mathrm{~min} 70 \% \mathrm{~A}$, and $2.50-4.00 \mathrm{~min} 85 \% \mathrm{~A}$. The flow rate is $0.20 \mathrm{ml} / \mathrm{min}$.

For all compounds, the MS instrument was operated in the positive ion electrospray ionization mode with multiple reaction monitoring. Sensitivity of the MRM was optimized by testing an infusion of $0.1 \mu \mathrm{g} / \mathrm{ml}$ EPI and IS in a mixture of acetonitrile and water $(1: 1, \mathrm{v} / \mathrm{v})$, respectively. Source conditions were typically as follows: capillary $3.0 \mathrm{KV}$, source temperature $110^{\circ} \mathrm{C}$ and desolvation temperature $180^{\circ} \mathrm{C}$. The cone and desolvation gas flows were 50 and $350 \mathrm{~L} / \mathrm{h}$, respectively. Nitrogen and argon were used as cone and collision gases, respectively. The collision gas was regulated at $2.34 \times 10^{-3}$ mbar. Multipliers were set to $650 \mathrm{~V}$ and the dwell time for each transition was $0.1 \mathrm{~s}$. The MRM transitions as well as the individual cone and collision energy voltages applied for the analysis are summarized in Table 1 .

All aspects of data acquisition were controlled using MassLynx ${ }^{\mathrm{TM}}$ NT 4.0 software with QuanLynx ${ }^{\mathrm{TM}}$ program (Waters). Peak areas of the chromatograms were integrated and the peak area ratios of the analyte/IS were calculated. A weight $1 / x^{2}$ linear regression was used to obtain calibration curve from standards. The regression equation of the calibration curve was then used to calculate the concentrations of method validation samples and clinical samples.

\subsection{Blood sample collection and preparation}

Blood samples (2-3 ml) came from cancer patients with malignant lymphoma, at XiYuan Hospital, Beijing. The drug was administered to patients by intravenous infusion at a dose

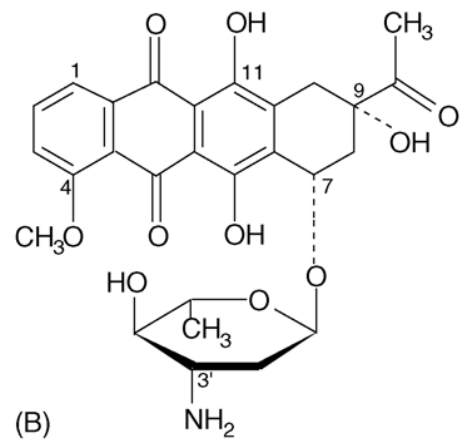

Fig. 1. Chemical structures of (A) EPI and (B) EPR (IS). 
Table 1

Mass transitions, cone and collision energy voltages for EPI and EPR (IS)

\begin{tabular}{llll}
\hline Compound & Molecular weight & Mass transitions & Cone voltage (V) \\
\hline EPI & 543.5 & $544 \rightarrow 130$ & 22 \\
& & $544 \rightarrow 397$ & 22 \\
EPR & 527.5 & $528 \rightarrow 321$ & 30 \\
& & $528 \rightarrow 363$ & 30 \\
\hline
\end{tabular}

of 50-60 mg. A sample was taken before the treatment of each patient, while a second sample was taken at a different time after the end of the treatment. The blood samples were collected in heparinized tubes, which were centrifuged (3000 rpm, Jouan MR 1822 , France) at $4{ }^{\circ} \mathrm{C}$ for $15 \mathrm{~min}$ to separate the plasma fractions. Each plasma sample was aliquoted $(0.2 \mathrm{ml})$ into polypropylene tubes and stored at $-20^{\circ} \mathrm{C}$ in the dark until analysis. The post-dose plasma samples were spiked with $100 \mathrm{ng} / \mathrm{ml}$ IS and briefly vortex-mixed before solid-phase extraction.

\subsection{Preparation of calibration standards}

Primary stock solutions of EPI and EPR (IS) $(1 \mathrm{mg} / \mathrm{ml})$ were prepared in methanol-water $(1: 1, \mathrm{v} / \mathrm{v})$, respectively. The stock solutions were stored in polypropylene tubes with screw caps and kept in a refrigerator at $4{ }^{\circ} \mathrm{C}$. Working standard solutions of EPI were prepared by diluting the primary solution with formate buffer $(20 \mathrm{mM}, \mathrm{pH} 2.9)$ to reach the concentrations of $800,400,160,80,40,20,8$ and $4 \mathrm{ng} / \mathrm{ml}$. In the same way, the working solution for internal standard $(800 \mathrm{ng} / \mathrm{ml})$ was prepared from the IS stock solution.

$0.2 \mathrm{ml}$ of drug-free human plasma were spiked $25 \mu \mathrm{l}$ of internal standard solution and $25 \mu \mathrm{l}$ of the appropriate working solutions of EPI to produce the following calibration levels: $0,0.5,1,2.5,5,10,20,50$ and $100 \mathrm{ng} / \mathrm{ml}$.

\subsection{Solid-phase extraction}

Each of the above-prepared calibration standards and plasma samples was loaded onto an Oasis HLB cartridge previously conditioned with $1 \mathrm{ml}$ of methanol and $1 \mathrm{ml}$ of deionized water. The cartridge was then washed sequentially with $1 \mathrm{ml}$ of $5 \%$ methanol (the first wash) and $1 \mathrm{ml}$ of $40 \%$ $(\mathrm{v} / \mathrm{v})$ methanol containing $2 \%$ ammonium hydroxide (the second wash). The analytes were eluted from the cartridge with $0.5 \mathrm{ml}$ of $0.5 \%$ formic acid methanol solution in $1.5 \mathrm{ml}$ Waters certified vials. The eluates were evaporated to dryness at $30^{\circ} \mathrm{C}$ under a gentle stream of nitrogen. The residue was reconstituted in $200 \mu \mathrm{l}$ of $15 \% \mathrm{ACN}$ in water and vortexed for $20 \mathrm{~s}$. A 10- $\mu \mathrm{l}$ aliquot was injected into the UPLC-MS/MS system.

\subsection{Method validation}

Validation samples at three different concentrations (i.e., $2.5,50.0$ and $100.0 \mathrm{ng} / \mathrm{ml}$ ) were prepared and analyzed to determine the extraction efficiency of the analyte and to evaluate the accuracy and precision of the method in plasma samples. The extraction recovery was determined by comparing the analyte/IS peak ratios of the spiked samples with those of unextracted neat solutions at the corresponding concentrations. The precision (repeatability) of the method was obtained by analyzing three replicates of validation samples at $2.5,50.0$ and $100.0 \mathrm{ng} / \mathrm{ml}$ on 3 days, respectively. The precision was determined by calculating the relative standard deviation (R.S.D.) for the repeated measurements. The limit of detection (LOD) and the limit of quantification (LOQ) were defined as the concentration of a signal-to-noise ratio of 3 and 10, respectively. Analyte stability was tested by analyzing validation samples at $100.0 \mathrm{ng} / \mathrm{ml}$ stored at $-20^{\circ} \mathrm{C}$ in the dark on every 7 days for a month.

\section{Results and discussion}

\subsection{Solid-phase extraction}

SPE has been proven to be an effective tool for selectively removing interferences, enabling sensitive, selective and robust LC-MS/MS analysis. Oasis HLB polymer was chosen for extracting the analytes from plasma in this study, due to its unmatched advantages to capture polar basic compounds and metabolites over silica-based sorbents [12]. Aliquots $(0.2 \mathrm{ml})$ of blank human plasma spiked with two levels of standard mixtures (i.e., 0.1 and $1.0 \mu \mathrm{g} / \mathrm{ml} \mathrm{EPI}$, added with $4.0 \mu \mathrm{g} / \mathrm{ml}$ EPR, respectively) were processed through all steps of the SPE to monitor the recoveries. The SPE method with two washes was employed for the extraction of the analytes in present study. In order to achieve high recoveries of the analytes and to remove the interferences as largely as possible, methanol concentrations in the second wash ranging from $30 \%$ to $50 \%$ were investigated. As a result, a good compromise was reached at $40 \%$ containing $2 \%$ ammonium hydroxide, and the clean extract can be obtained with high recoveries. In addition, several solutions were tested as the elution solvent, including methanol, $0.5 \%$ trifluoroacetic acid (TFA) in methanol, $0.5 \%$ formic acid in methanol, $0.5 \%$ formic acid in ACN and $90 \% \mathrm{ACN}$ in formate buffer $(40 \mathrm{mM}, \mathrm{pH} 2.9)$. The results showed that large elution volume $(>1 \mathrm{ml})$ of methanol or $0.5 \%$ TFA in methanol or $0.5 \%$ formic acid in $\mathrm{ACN}$ was needed to obtain complete elution of the analytes of interest. $0.5 \mathrm{ml}$ of $0.5 \%$ formic acid in methanol or $90 \% \mathrm{ACN}$ in formate buffer $(40 \mathrm{mM}, \mathrm{pH} 2.9)$ 

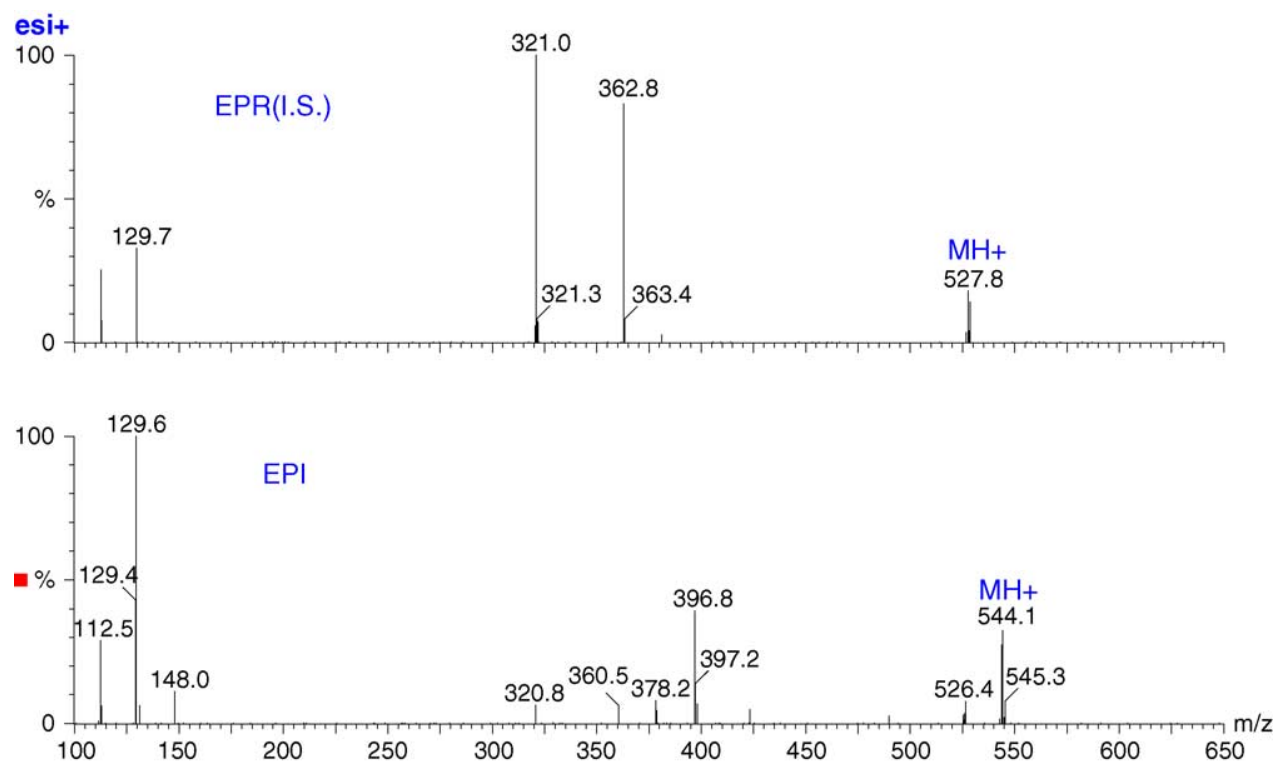

Fig. 2. Product ion mass spectra of EPI and EPR (IS).

could elute the analytes with satisfactory recoveries. But, the evaporation time of the latter is much longer than the former. Therefore, $0.5 \mathrm{ml}$ of $0.5 \%$ formic acid in methanol was chosen as the elution solvent to avoid time consumption. After the overall extraction process (including two washes, evaporation and reconstitution), the absolute recoveries were $84.2 \%$ for $4 \mu \mathrm{g} / \mathrm{ml} \mathrm{EPR}, 83.4 \%$ and $86.9 \%$ for $0.1 \mu \mathrm{g} / \mathrm{ml}$ and $1 \mu \mathrm{g} / \mathrm{ml}$ EPI, respectively.
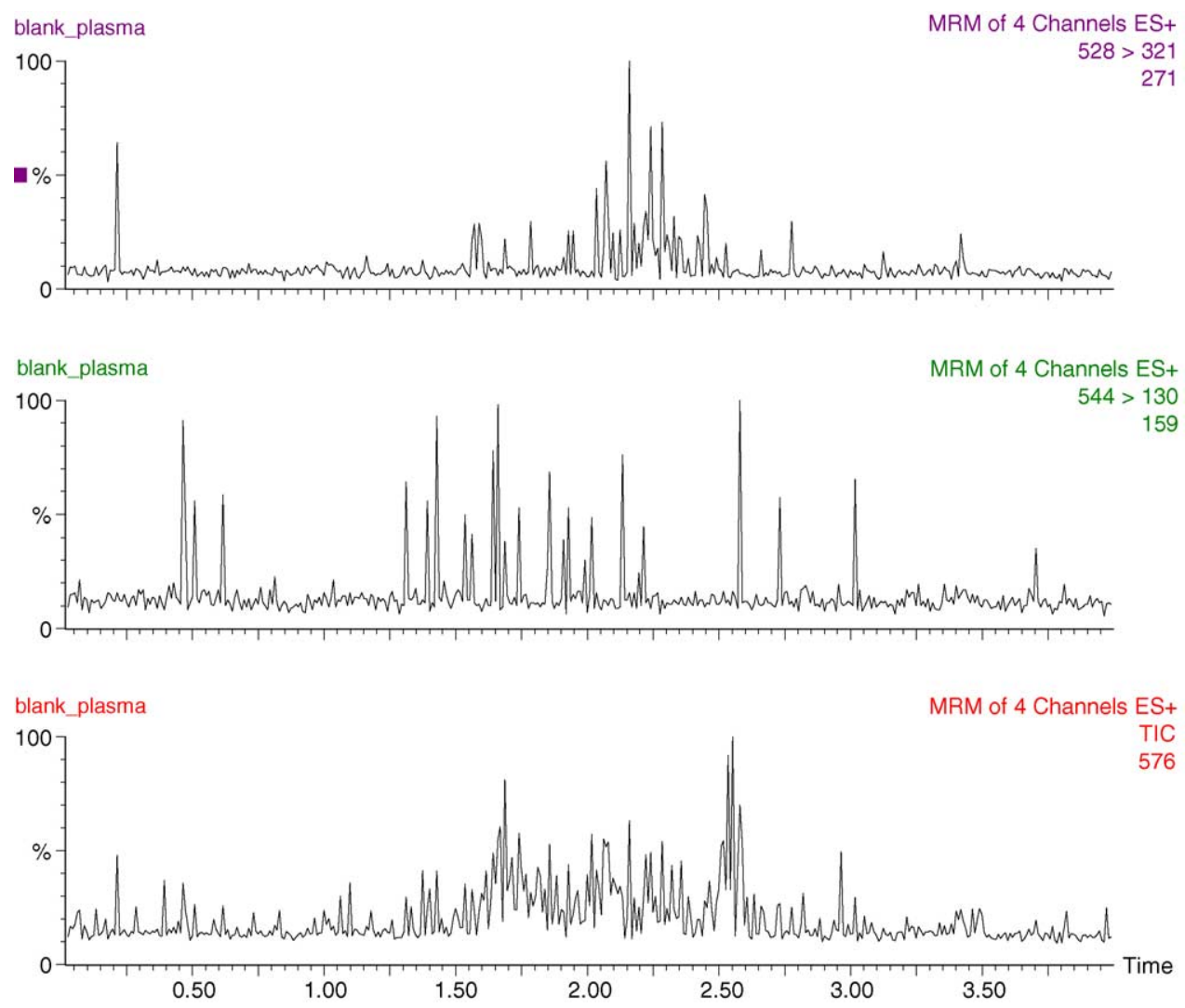

Fig. 3. TIC and MRM UPLC-MS/MS chromatograms of blank human plasma from a patient prior to treatment. Conditions are the same as described in the text. 


\subsection{Mass spectra}

At the optimum conditions as shown in Table 1, the electrospray ionization of EPI and EPR produced the abundant $\mathrm{MH}+$ at mass-to-charge ratio $(\mathrm{m} / \mathrm{z}) 544$ and 528, respectively. The corresponding $\mathrm{MH}+$ ions from EPI and EPR were selected as the precursor ions and subsequently fragmented in MS/MS mode. Fig. 2 shows the product ion mass spectra (mass range $\mathrm{m} / \mathrm{z}, 100-650$ ) of each compound. It can be seen obviously that there are two predominant product ions at $\mathrm{m} / \mathrm{z} 397$ (the loss of amino sugar moiety from $\mathrm{MH}+$ ion) and 130 (the loss of $\mathrm{H}_{2} \mathrm{O}$ from the protonated ion of amino sugar, $\mathrm{m} / \mathrm{z}$ 148) for EPI, and 363 (the loss of amino sugar moiety and $\mathrm{H}_{2} \mathrm{O}$ from $\mathrm{MH}+$ ion) and 321 (the loss of $\left[\mathrm{CO}+\mathrm{CH}_{2}\right]$ in the 9-acetyl chain from $\mathrm{m} / \mathrm{z} 363$ product ion) for EPR, respectively. The analytes were identified and determined by using MRM with the specific transitions. Though the metabolite EPL could not be obtained as a pure standard, it was also monitored in this study. EPL, differing from EPI by the substitution of carbonyl (i.e., $-\mathrm{C}=\mathrm{O}$ ) with $-\mathrm{OH}$ in the 9-acetyl chain, generates the protonated molecular ion at $m / z$ 546.5 [1,9]. The MRM transition $546 \rightarrow 399$ for EPL was followed based on the cleavage of its parent drug.
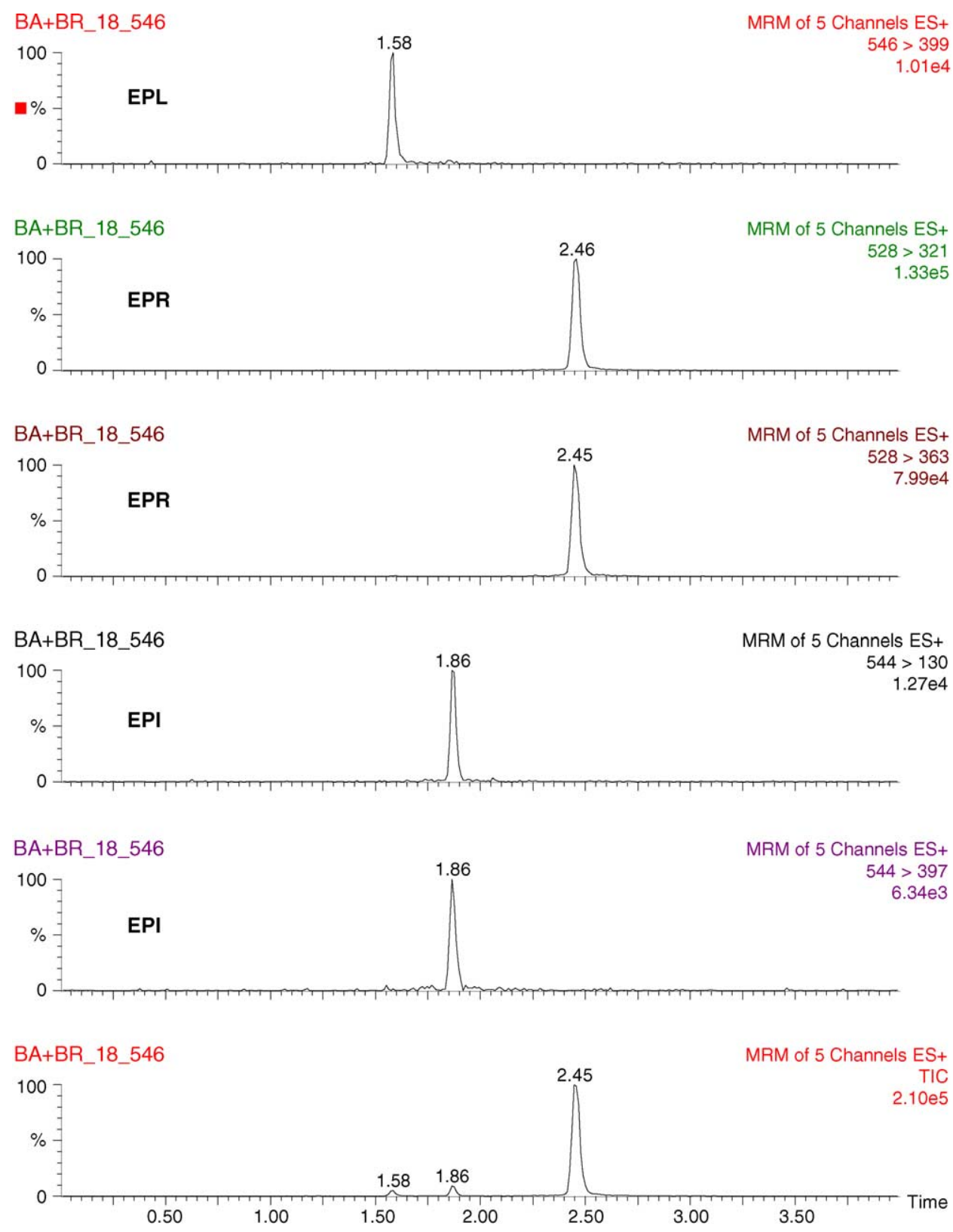

Fig. 4. TIC and MRM UPLC-MS/MS chromatograms of human plasma (spiked with $100.0 \mathrm{ng} / \mathrm{ml}$ IS) from the same patient in Fig. 3 obtained $8 \mathrm{~h}$ after intravenous administration of EPI $(60 \mathrm{mg})$. Conditions are the same as described in the text. 
Table 2

Validation data of the method

\begin{tabular}{|c|c|c|c|c|c|}
\hline \multirow{2}{*}{$\begin{array}{l}\text { Concentration } \\
\text { added }(\mathrm{ng} / \mathrm{ml})\end{array}$} & \multirow{2}{*}{$\begin{array}{l}\text { Extraction } \\
\text { recovery }(\%)\end{array}$} & \multirow{2}{*}{$\begin{array}{l}\text { Intra-day precision } \\
(n=3) \text { R.S.D. }(\%)\end{array}$} & \multicolumn{3}{|l|}{ Inter-day study $(n=3)$} \\
\hline & & & Mean concentration found $(\mathrm{ng} / \mathrm{ml})$ & Precision RSD (\%) & Relative mean error $(\%)$ \\
\hline 2.5 & 101.2 & 4.5 & 2.3 & 11.7 & -8.0 \\
\hline 50.0 & 89.4 & 5.2 & 50.3 & 8.0 & +0.6 \\
\hline 100.0 & 91.4 & 3.5 & 101.2 & 6.1 & +1.2 \\
\hline
\end{tabular}

Table 3

Concentration of EPI found in plasma samples from treated patients

\begin{tabular}{|c|c|c|c|c|}
\hline Subject (no.) & Treatment (mg) & Time of post-administration (h) & EPI (ng/ml) (mean, $n=3)$ & R.S.D. (\%) \\
\hline 1 & 60 & 22 & 1.0 & 3.85 \\
\hline 2 & 60 & 8 & 5.8 & 2.89 \\
\hline 3 & 50 & 5 & 9.5 & 4.84 \\
\hline
\end{tabular}

\subsection{UPLC-MS/MS analysis}

UPLC method was operated on the AcQuity UPLC ${ }^{\mathrm{TM}}$ BEH C $_{18}(1.7 \mu \mathrm{m})$ column and optimized to achieve the best separation and retention for the analytes. With the increase of acetonitrile the retention for the analytes decreases, as predicted by reversed phase mechanism. The separation was performed under gradient conditions to reduce the total run time. The addition of formic acid $(0.1 \%, \mathrm{v} / \mathrm{v})$ was required to improve the ionization efficiency. Figs. 3 and 4 are the representative total ion current (TIC) and MRM chromatograms obtained from the analysis of clinical samples. Fig. 3 displays the chromatogram of a blank plasma sample taken from the patient prior to treatment, and Fig. 4 depicts the chromatogram of a plasma sample (spiked with $100.0 \mathrm{ng} / \mathrm{ml}$ IS) from the same patient after treatment with EPI. Both EPI and EPL were detected with excellent separation and peak shapes. All the compounds including the IS were rapidly eluted (less than $4 \mathrm{~min}$ ), and no interfering peaks were observed in the blank sample and in all samples examined. Moreover, the UPLC column demonstrated excellent stability as shown by very stable retention time $\left(t_{\mathrm{R}}\right)$ and unchanged peak shape for both EPI and IS. After about 300 injections of extracted samples, the EPI retention changed from 1.88 to $1.86 \mathrm{~min}$, and no significant increase in column back pressure was found. SPE method applied (Section 3.1) accomplishes satisfactory clean up of plasma samples, so that the latter could be assayed by the UPLC-MS/MS.

Calibration curve was constructed for EPI by plotting the peak area ratios (analyte/IS) against the concentration. A weighted $\left(1 / x^{2}\right)$ linear regression line was applied. Linear responses were obtained for EPI over the range $0.50-100 \mathrm{ng} / \mathrm{ml}$ with a regression coefficient of 0.998 , and intercepted the $y$ axis close to the origin. The limit of quantification and the limit of detection were 0.5 and $0.1 \mathrm{ng} / \mathrm{ml}$, respectively.

\subsection{Method validation}

The method validation data (extraction efficiency, intraand inter-day variation) for the analyte are summarized in
Table 2. The extraction recoveries ranged from 89.4 to $101.2 \%$, while the precision values ranged from 3.5 to $11.7 \%$ over the three concentrations (i.e., $2.5,50$ and $100 \mathrm{ng} / \mathrm{ml}$ ) evaluated during 3 days. The analyte stability test showed that validation samples stored in a freezer at $-20^{\circ} \mathrm{C}$ remained stable at least for 1 month. These results indicated that the proposed method has an acceptable accuracy and precision.

\subsection{Application to clinical samples}

Using the methodology established above, plasma samples collected in patients administered EPI intravenously were analyzed (Table 3). EPL was detected in all samples as expected. A typical chromatogram is displayed in Fig. 4. The results demonstrate that the method is usable and applicable to the detection and quantification of EPI and its metabolite. In comparison with the available LC-MS method for analysis of EPI in serum [9], the LOD and LOQ of this method are lowered about 10 and 5-fold, while the amount of plasma required is less. This sensitivity increase results not only from the improvements in the sensitivity and selectivity provided by incorporating MS/MS detection, but also from the benefits provided by UPLC: high efficiency due to limited diffusion. Furthermore, the run time of the analysis is shorter than those of published methods, allowing increased sample throughput for routine purposes.

\section{Conclusions}

A simple, ultra-fast and accurate method based on UPLC-MS/MS has been shown to be suitable for clinical plasma samples of small volumes $(0.2 \mathrm{ml})$ with low concentrations of EPI. The limits of detection and quantification for epirubicin were 0.10 and $0.50 \mathrm{ng} / \mathrm{ml}$, respectively. The sensitivity of the current method could be further improved by reducing sample reconstitution volume and increasing injection volume. Moreover, the sample throughput could be increased drastically because of the short analysis time (within $4 \mathrm{~min}$ ). The method has been successfully applied to 
plasma samples from patients receiving EPI chemotherapy. As a result of its applicability in clinical practice, it is very useful for therapeutic drug monitoring and for clinical and pharmacokinetic studies of epirubicin.

\section{Acknowledgement}

The authors would like to thank Waters Corporation in China for the kind support of the UPLC-MS/MS instrument.

\section{References}

[1] G. Zagotto, B. Gatto, S. Moro, C. Sissi, M. Palumbo, J. Chromatogr. B 764 (2001) 161

[2] J.L. Quiles, J.R. Huertas, M. Battino, J. Mataix, M.C. RamírezTortosa, Toxicology 180 (2002) 79.
[3] S.A. Laurie, D. Logan, B.R. Markman, J.A. Mackay, W.K. Evans, Lung Cancer Disease Site Group of Cancer Care Ontario's Program in Evidence-based Care, Lung Cancer 43 (2004) 223.

[4] N.J. Reinhoud, U.R. Tjaden, H. Irth, J. van der Greef, J. Chromatogr. 574 (1992) 327.

[5] R. Ricciarello, S. Pichini, R. Pacifici, I. Altieri, M. Pellegrini, A. Fattorossi, P. Zuccaro, J. Chromatogr. B 707 (1998) 219.

[6] G. Nicholls, B.J. Clark, J.E. Brown, J. Pharm. Biomed. Anal. 10 (1992) 949.

[7] A. Rudolphi, S. Vielhauer, K.-S. Boos, D. Seidel, I.-M. Bathge, H. Berger, J. Pharm. Biomed. Anal. 13 (1995) 615.

[8] I.K. Barker, S.M. Crawford, A.F. Fell, J. Chromatogr. B 681 (1996) 323.

[9] F. Lachâtre, P. Marquet, S. Ragot, J.M. Gaulier, P. Cardot, J.L. Dupuy, J. Chromatogr. B 738 (2000) 281.

[10] J. de Jong, A. Bast, W.J.F. van der Vijgh, TrAC Trends Anal. Chem. 12 (1993) 422.

[11] P.M. Loadman, C.R. Calabrese, J. Chromatogr. B 764 (2001) 193.

[12] Y.-F. Cheng, U.D. Neue, L. Bean, J. Chromatogr. A 828 (1998) 273. 